\title{
$P-19$
}

\section{Difference of serum uric acid and its related clinical characteristics in Chinese CKD patients compared with those of Japanese.}

\author{
Sun $\mathrm{Na}^{1)} \quad \mathrm{Xu} \mathrm{Ning}^{1)}$ \\ Takanori Kumagai ${ }^{2}$ \\ Shigeru Shibata ${ }^{2}$ \\ Shen Zhong Yang ${ }^{3)}$ \\ Chang Wen $\mathrm{Xiu}^{1)}$
}

\author{
Yuan Wei, ${ }^{1,2}$ \\ Yoshifuru Tamura ${ }^{2}$ \\ Yoshihide Fujigaki ${ }^{2}$ \\ Shunya Uchida ${ }^{2}$
}

Background. The significance of the impact of serum uric acid (SUA) on the CKD progression yet to be widely recognized in China, thus intervention to treat hyperuricemia is unlikely adequately achieved. The current situation of SUA and its related clinical characteristics were investigated and compared with those of Japanese.

Methods. Stage 3 to 4 CKD participants in China consisted of 224 patients who were followed-up in Tianjin First Center Hospital (age 57 \pm 14 , male 138: female 86). Japanese Stage 3 to 4 CKD cohort included the patients in Teikyo University Hospital $(n=803$, age $62 \pm 13$. male 501 : female 302$)$. The baseline SUA was compared between 2 countries and multivariate linear regression analysis was performed to examine the influence of other baseline covariates on SUA. The significant difference of the country was tested for an interaction effect.

Results. Mean SUA in China was significantly higher than those in Japan $(6.99 \pm 1.82$ vs. $6.48 \pm$ $1.43 \mathrm{mg} / \mathrm{dL}, p<0.001)$. Baseline eGFR was lower in China ( $36 \pm 13$ vs. $\left.41 \pm 13 \mathrm{ml} / \mathrm{min} / 1.73 \mathrm{~m}^{2}, p<0.001\right)$. Systolic blood pressure was higher and hemoglobin was lower in China. In Japanese CKD patients, SUA was significantly associated with male, younger age, lower eGFR. higher BMI, use of diuretics and less use of urate-lowering drugs $\left(R^{2}=0.18, p<0.001\right)$. In contrast, SUA of Chinese CKD patients was associated with male, higher BMI and lower eGFR $\left(R^{2}=0.11\right.$, $p=0.002)$. The interaction effect was observed only between BMI and country, and indeed, the slope between SUA and BMI was steeper in Chinese by $\operatorname{ANCOVA}(p=0.010)$.

Conclusions. Chinese CKD patients remain at higher risk of CKD progression than Japanese CKD patients. In particular, urate-lowering drug was less frequently used despite high SUA compared with Japan. Education of total care for inhibiting CKD progression including hyperuricemia is needed in China. Higher BMI more strongly influences SUA in Chinese than Japanese CKD patients, the ethnic difference of which warrants further investigation.

1 ) Department of Nephrology, Tianjin First Center Hospital, Tianjin, China

2 ) Department of Internal Medicine, Teikyo University School of Medicine, Tokyo, Japan

3 ) Department of Organ Transplantation, Tianjin First Center Hospital, Tianjin, China 